\title{
Simultaneous Bistability of a Qubit and Resonator in Circuit Quantum Electrodynamics
}

\author{
Th. K. Mavrogordatos, ${ }^{1, *}$ G. Tancredi, ${ }^{2}$ M. Elliott, ${ }^{3}$ M. J. Peterer, ${ }^{2}$ A. Patterson, ${ }^{2}$ \\ J. Rahamim, ${ }^{2}$ P. J. Leek, ${ }^{2}$ E. Ginossar, ${ }^{3}$ and M. H. Szymańska ${ }^{1}$ \\ ${ }^{1}$ Department of Physics and Astronomy, University College London, Gower Street, London WC1E 6BT, United Kingdom \\ ${ }^{2}$ Clarendon Laboratory, University of Oxford, Parks Road, Oxford OX1 3PU, United Kingdom \\ ${ }^{3}$ Advanced Technology Institute and Department of Physics, University of Surrey, Guildford GU2 7XH, United Kingdom
}

(Received 24 June 2016; revised manuscript received 30 November 2016; published 24 January 2017)

\begin{abstract}
We explore the joint activated dynamics exhibited by two quantum degrees of freedom: a cavity mode oscillator which is strongly coupled to a superconducting qubit in the strongly coherently driven dispersive regime. Dynamical simulations and complementary measurements show a range of parameters where both the cavity and the qubit exhibit sudden simultaneous switching between two metastable states. This manifests in ensemble averaged amplitudes of both the cavity and qubit exhibiting a partial coherent cancellation. Transmission measurements of driven microwave cavities coupled to transmon qubits show detailed features which agree with the theory in the regime of simultaneous switching.
\end{abstract}

DOI: 10.1103/PhysRevLett.118.040402

The generalized Jaynes-Cummings (GJC) model provides a simple basis for describing the interactions between a quantized electromagnetic field and multilevel atoms. Its nonlinearity lies at the heart of cavity quantum electrodynamics (cavity QED), where natural atoms are coupled to cavity photons [1], and circuit quantum electrodynamics (circuit QED), where artificial atoms are coupled to resonators of various dimensionalities [2-6]. The JaynesCummings (JC) interaction also emerges in areas of current interest such as optomechanics in the linearized regime [7] and in the Bose-Hubbard model [8]. There is a large body of work on the resonant and strong-coupling regime of the driven-dissipative JC oscillator $[9,10]$, where driving induces a dynamical Rabi splitting [11,12]. The high excitation strong-dispersive regime is also of great interest, for example, in the context of amplifiers [13], squeezing associated with the parametric oscillator [14], and the implementation of qubit readout schemes [15-18]. In this context, fluctuation-induced switching between metastable states in the driven-dissipative GJC system, which involves two quantum degrees of freedom, has not been directly studied, but the theory of quantum activation motivates the interest in this scenario [19-25].

In this Letter, we demonstrate that in a nonlinear intermediate driving regime of circuit QED, the system dynamics exhibits a simultaneous bistability of the qubit and resonator. We support this claim both theoretically, with analytical and numerical results, as well as with

Published by the American Physical Society under the terms of the Creative Commons Attribution 4.0 International license. Further distribution of this work must maintain attribution to the author(s) and the published article's title, journal citation, and DOI. experimental measurements obtained from a circuit QED device, consisting of a transmon qubit coupled to a $3 \mathrm{D}$ microwave cavity [26]. In particular we report the following findings. (1) The switching process occurs simultaneously for the two coupled quantum oscillators. (2) The ensemble averaged amplitudes of both cavity and qubit exhibit a coherent partial cancellation. Such cancellation, predicted theoretically for a single nonlinear mode by Drummond and Walls in Ref. [27], is here verified experimentally and shown to occur for both coupled oscillators. (3) The Duffing oscillator model with one quantum degree of freedom is not sufficient to account for the observed cavity nonlinearity when the two coupled quantum degrees of freedom are involved in the switching process. We also note that the JC bistable semiclassical intracavity amplitude $|\alpha|_{\mathrm{ss}}^{2}$ (with $\alpha=\langle a\rangle$ ) in the steady state, plotted in Fig. 1(a), does not show any coherent cancellation feature, nor does the bistable average atomic inversion $\zeta_{\mathrm{ss}}=\left\langle\sigma_{z}\right\rangle_{\mathrm{ss}}$ shown in Fig. 1(b). Instead, both curves are skewed Lorentzians with the position of their peaks approaching the bare cavity frequency for increasing drive. By increasing the drive strength beyond what is shown in Fig. 1(a), one finds a critical point in the phase space between bistable and linear behavior, lying on the line where the frequency of the drive equals the bare cavity resonance frequency. Beyond that point, the system behaves as a linear oscillator, in contrast to the corresponding Duffing oscillator [17], and exhibits no bistability.

Theoretical models. - In order to develop a comprehensive understanding of the system response, we will consider several different theoretical models: (i) a multilevel transmon-cavity GJC model-the most complete in the context of superconducting devices, (ii) a two-level atom-cavity JC model which is universal to many strong light-matter coupling scenarios, and (iii) a simplified dressed-cavity 


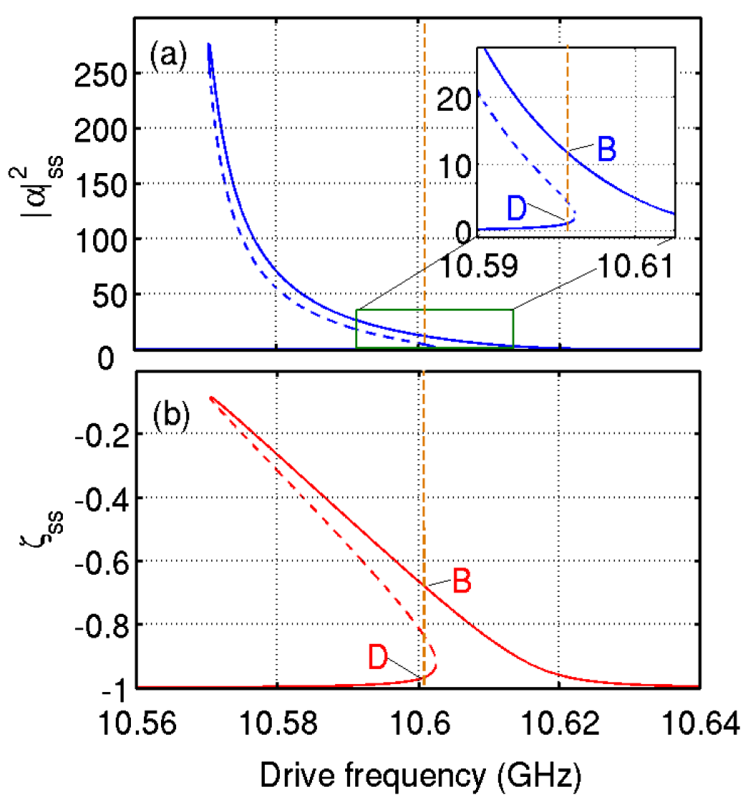

FIG. 1. Maxwell-Bloch steady-state bistability of the JC oscillator in the dispersive regime [6] for the coupling strength to detuning ratio $g / \delta=0.14$, the drive amplitude to photon loss ratio $\varepsilon_{d} /(2 \kappa)=25 / 3$, and the photon loss to spontaneous emission ratio $2 \kappa / \gamma=12$. Here, $B(D)$ denote the bright (dim) semiclassical states. (a) Intracavity field amplitude in the steady state (with the region of interest zoomed in the inset). (b) The corresponding atomic inversion $\zeta=\left\langle\sigma_{z}\right\rangle$. The broken orange line indicates the driving frequency at $10.6005 \mathrm{GHz}$, and the dashed lines in each curve depict the unstable branch.

Duffing oscillator approximation. To complement the numerical simulations of the three models, we additionally compare the results for the cavity transmission with those coming from an analytical formula, obtained by modeling the transmon itself as a nonlinear oscillator.

We will now define the system Hamiltonians associated with models (i)-(iii). When one cavity field mode of frequency $\omega_{c}$ (with corresponding photon annihilation and creation operators $a$ and $a^{\dagger}$, respectively) is coupled to a multilevel system with unperturbed states $|n\rangle$, the coherently driven GJC Hamiltonian can be written as (setting $\hbar=1$ ) [2]

$$
\begin{aligned}
H_{\mathrm{GJC}}^{(\mathrm{i})}= & \omega_{c} a^{\dagger} a+\sum_{n} \omega_{n}|n\rangle\left\langle n\left|+\sum_{m, n} g_{m n}\right| m\right\rangle\langle n|\left(a+a^{\dagger}\right) \\
& +i \varepsilon_{d}\left(a^{\dagger} e^{-i \omega_{d} t}-a e^{i \omega_{d} t}\right)
\end{aligned}
$$

where $\varepsilon_{d}$ is the strength of a monochromatic external field with frequency $\omega_{d}$ driving the cavity mode. The sum in the third term describes the interaction and is customarily modified to $\sum_{m} g_{m, m+1}\left(|m\rangle\langle m+1| a^{\dagger}+\right.$ H.c. $)$ in the rotating wave approximation (RWA). The interaction energies in the RWA have the approximate form $g_{m n} \approx g \sqrt{m+1} \delta_{m+1, n}$, with $g$ being the dipole coupling strength. Depending on the range of $n$ and the form of $\omega_{n}$, we can distinguish the two-level atom $\left(n=1, m=0, \omega_{n}=\omega_{q}\right)$-the JC model- from a transmon $\left(n=1,2, \ldots, N_{\max }, \omega_{n}=\omega(n)\right)$ - the GJC model. The JC model in the RWA reads

$$
H_{\mathrm{JC}}^{(\mathrm{ii})}=\omega_{c} a^{\dagger} a+\frac{1}{2} \omega_{q} \sigma_{z}+g\left(a^{\dagger} \sigma_{-}+a \sigma_{+}\right),
$$

with $\sigma_{ \pm}$the raising (lowering) pseudospin operators and $\sigma_{z}=2 \sigma_{+} \sigma_{-}-1$ the inversion operator. In the presence of dissipation, the cavity mode is damped at a rate $2 \kappa$ [28] while spontaneous emission is present at a rate $\gamma$ for a qubit dephased at a rate $\gamma_{\phi}$. It is possible to approximate the Hamiltonian of Eq. (2) further in the strongly dispersive regime, defined by the strong detuning $\delta=\left|\omega_{c}-\omega_{q}\right| \gg g$ between the two coupled oscillators. Under an appropriate decoupling transformation [21,29], $H_{\mathrm{JC}}$ can be recast in the form $H_{\mathrm{JC}}^{\prime}=\omega_{c} a^{\dagger} a+(1 / 2)\left(\omega_{c}-\Delta\right) \sigma_{z}$, involving the operator $\Delta=\sqrt{\delta^{2}+4 g^{2} \mathcal{N}}$ where $\mathcal{N}=a^{\dagger} a+\sigma_{+} \sigma_{-}$is the operator of the total number of excitations $N$. In the dispersive regime, provided that $N / N_{\text {crit }} \ll 1$ [30], we can expand up to the quartic order in the field variables. After normal ordering, we obtain the following dressed-cavity Duffing oscillator Hamiltonian

$H_{D}^{(\mathrm{iii})}=\left(\omega_{c}+\frac{g^{4}}{\delta^{3}}-\frac{g^{2}}{\delta} \sigma_{z}+2 \frac{g^{4}}{\delta^{3}} \sigma_{z}\right) a^{\dagger} a+\frac{g^{4}}{\delta^{3}} \sigma_{z} a^{\dagger 2} a^{2}$,

where setting $\sigma_{z}=-1$ is a justifiable approximation for low enough driving amplitudes, yielding a bistable quantum Duffing oscillator [35]. The third term in the parentheses is the leading-order term $\chi_{c}^{(0)}=\left(g^{2} / \delta\right) \sigma_{z}$ which is the familiar Stark shift [36] and provides a valuable tool for qubit readout (with $\left|\chi_{c}^{(0)}\right| \gg \kappa$ ). Here, in the bifurcating dispersive region we are studying, the following hierarchy of scales applies [17]: $\gamma, \gamma_{\phi} \ll 2 \kappa \ll \varepsilon_{d} \lesssim g^{2} / \delta \ll g<\delta \ll \omega_{c}$. The intracavity excitation number is of the order of $N_{\text {crit }}$, where this perturbation expansion is not strictly valid. However, as we will see later, it gives qualitatively meaningful results.

Having defined the different model Hamiltonians under consideration, we now evolve the corresponding master equations (MEs) in the finite Hilbert state basis numerically, starting from a Fock state of zero photons and the qubit in the ground state, until it reaches a steady state. Note that the steady state obtained is independent of the choice of the initial conditions.

Activated dynamics in the dispersive regime.-Driving the system beyond the low power regime has a profound effect on the response. We illustrate this fact in Fig. 2, where we depict the qubit inversion $\left\langle\sigma_{z}\right\rangle$ in Fig. 2(a), the photon cavity number $\left\langle n_{p}\right\rangle=\left\langle a^{\dagger} a\right\rangle$ in Fig. 2(b), alongside their associated cavity quasidistribution function in Fig. 2(d), employing the exact ME simulations [Fig. 2(d)] and single quantum trajectories from the stochastic Schrödinger equations (SSEs) using the second-order weak scheme in the diffusive approximation [37,38] [Figs. 2(a) and 2(b)]. 


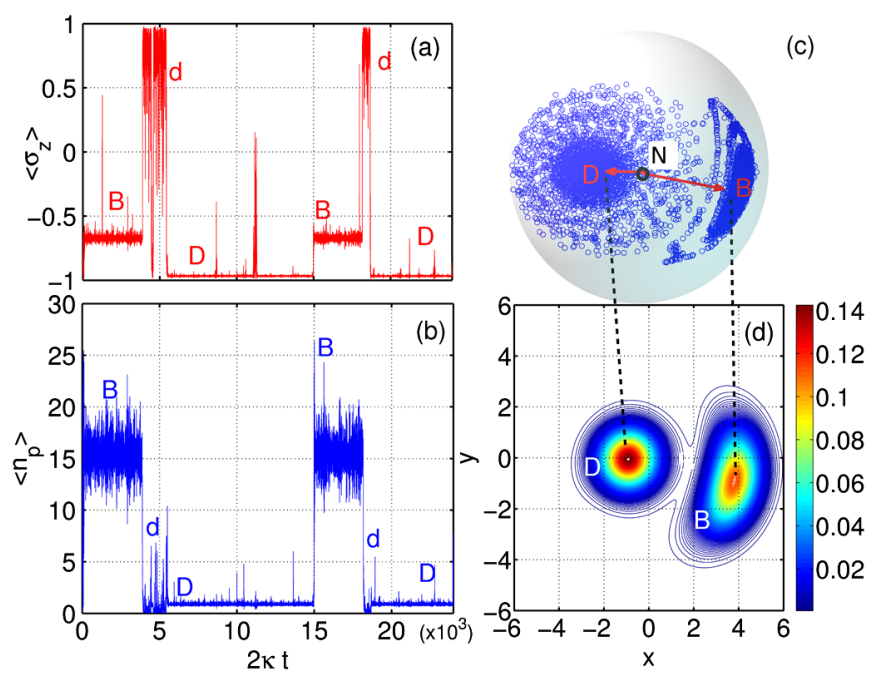

FIG. 2. Quantum-activated simultaneous cavity and qubit switching illustrated using the JC model in the dispersive regime for $g / \delta=0.14,3 \varepsilon_{d} /(2 \kappa)=25,2 \kappa / \gamma=12$, and $N_{\text {crit }} \simeq 13$. Here, $B(D)$ denote the bright (dim) metastable states and $d$ denotes the nonclassical dark state. (a) Atomic population inversion $\left\langle\sigma_{z}\right\rangle$. (b) The accompanying intracavity photons $\left\langle n_{p}\right\rangle=\left\langle a^{\dagger} a\right\rangle$ as a function of the dimensionless time $2 \kappa t$ for a single quantum trajectory. The trajectories in (a) and (b) depict simultaneous switching between the bright $(B)$ and $\operatorname{dim}(D)$ states. (c) Illustration depicting the two metastable state distributions in the Bloch sphere (as viewed from the north pole indicated by the letter $N)$. Data points corresponding to the dark state are omitted for clarity. The red arrows point to the two metastable states $(B$ and $D$ ). (d) Contour plot of the joint quasidistribution function $Q(x+i y)$ for $f_{d}=\omega_{d} / 2 \pi=10.6005 \mathrm{GHz}$, as indicated in Fig. 1, showing two peaks corresponding to two semicoherent states, indicating the presence of cavity bimodality.

Single quantum trajectories, corresponding to the unravelling of the ME for the JC Hamiltonian, depict the switching between the two metastable semiclassical states as a result of quantum fluctuations. The switching occurs simultaneously for the qubit [Fig. 2(a)] and the cavity [Fig. 2(b)]. The corresponding photon histogram shows quasi-Poissonian statistics obeyed by the two metastable states, one with mean photon occupation of the order of $N_{\text {crit }}$ (called a bright state), one with mean occupation of about a photon (called a dim state), as well as the distribution of a nonclassical (called a dark) state (for more details, see Ref. [39]). In Fig. 2(c), we draw a sketch illustrating the qubit distribution in the steady state, as viewed from the north pole of the Bloch sphere, for a single quantum trajectory. Red arrows point to the two semiclassical qubit states, corresponding to the two metastable quasicoherent cavity states [depicted by color contour plots in Fig. 2(d)] between which quantumactivated switching takes place. The $Q$ function plot in Fig. 2(d) also shows the position in the phase space of the coherently cancelling states. The equal height of the $Q$ function peaks indicates the boundary of a first-order dissipative quantum phase transition $[8,42]$. This transition is marked by the switching rates to the bright and to the dim state being of the same order of magnitude $[8,19]$. In our case, as well as in the exact photon statistics section of Ref. [27], switching is induced by quantum fluctuations only, as the thermal bath to which the system is coupled is at zero temperature. The trajectories depicted for illustration in Fig. 2 evidence sudden simultaneous jumps. Bistability and synchronization were studied for the two-level Rabi model in [43]. Note that, in contrast to the cavity field and $\left|\left\langle\sigma_{-}\right\rangle\right|$, the exact ME results for the photon number and $\left\langle\sigma_{z}\right\rangle$ show no coherent cancellation in the steady-state response. The mean-field behavior depicted in Fig. 1 shows further that the coherent cancellation is purely a quantum effect at zero temperature, occurring when forming the ensemble averaged quantities, and is already present in the most approximate dressed-cavity Duffing model, even if the qubit is unmonitored.

The nonlinear resonator transmission line shape.-In Fig. 3(a), we compare theoretical and experimental transmission amplitudes of a 3D cavity with embedded transmon (device $D_{1}$, details in Ref. [39]) for different driving strengths. We observe that, as the driving power is increased, the experimental cavity line shape develops nonlinear features and a coherent cancellation dip appears. We find perfect agreement with the GJC model. In Fig. 3(b), we show the cavity transmission for the intermediate drive power of $-46 \mathrm{dBm}$ for all the models discussed. We observe that the JC model predicts the split of the main peak at the correct position, as opposed to its Duffing reduction, yet fails to capture the position of the dip emerging at a lower frequency. The GJC model with four transmon levels can resolve all the details necessary for a quantitative comparison and provides indeed the most complete description of the cavity nonlinearity.

The behavior of the GJC oscillator depends strongly on the drive strength and frequency, and their relation to the coupling and dissipation rates $[14,17]$. We find theoretically that the coherent cancellation dip in transmission, discovered by Drummond and Walls for the Duffing oscillator [27], appears in the dispersive response of both the cavity and the qubit within the full nonlinearity of the JC model, where the departure from the mean-field predictions is appreciable and the Duffing oscillator approximation is no longer valid. For the Duffing oscillator, the dip is present in the first moment of the field operator $|\langle a\rangle|$ calculated using the generalized $P$ representation [27]. It is purely a phase effect as the dip does not appear in the number of intracavity photons in the steady state $\left\langle n_{p}\right\rangle=\left\langle a^{\dagger} a\right\rangle$. The coherent cancellation dip appears as well in the qubit projection $\left|\left\langle\sigma_{-}\right\rangle\right|$(see Ref. [39] for more details). The presence of this dip in the cavity response has also been observed in our experimental measurements, which depict the development of nonlinearity for increasing drive strengths within the region of bistability. Similar cancellation effects appear also in classical dissipative systems out of equilibrium, in the presence of thermal fluctuations [27]. The observed dip, appearing 

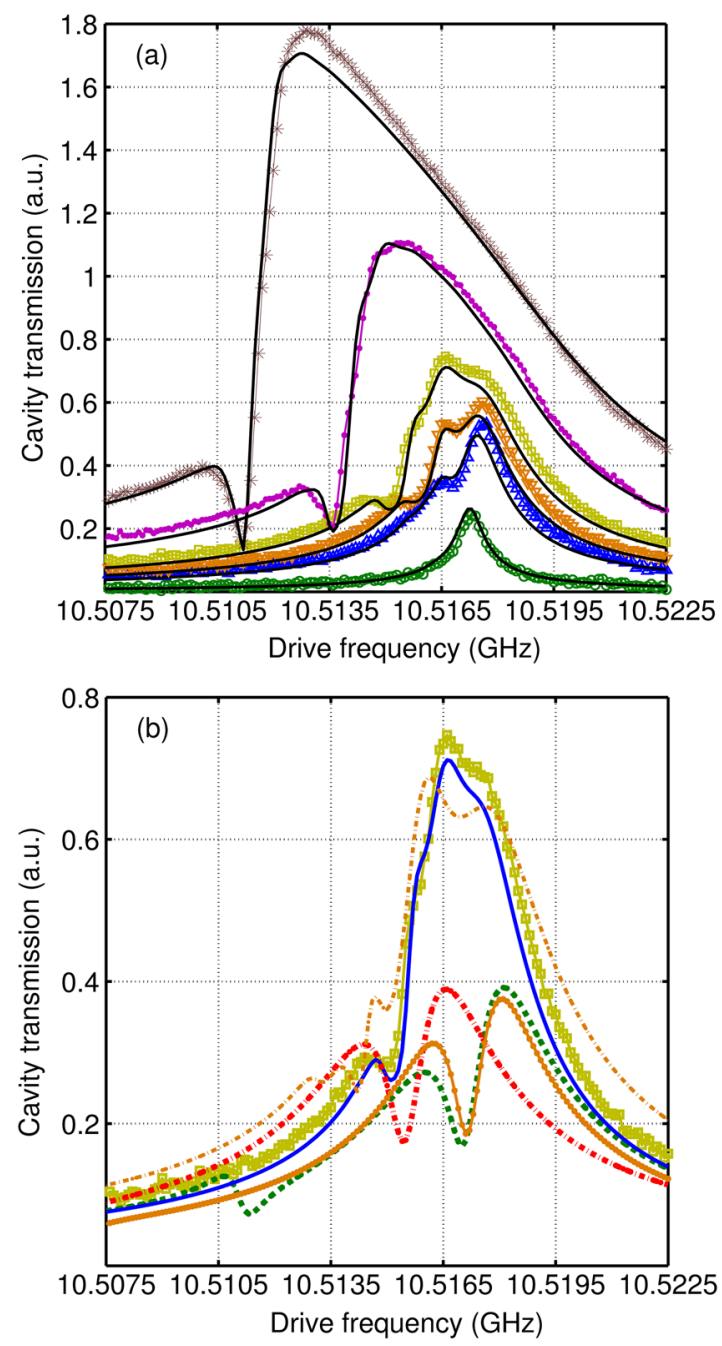

FIG. 3. Theory and experiment showing the cavity nonlinearity for increasing drive strength. (a) The experimentally measured transmission amplitude (in patterned lines) of the device $D_{1}$, consisting of a cavity resonator with bare frequency $f_{c}=$ $\omega_{c} / 2 \pi=10.426 \mathrm{GHz}$ coupled to a transmon qubit with lower transition frequency $f_{q}=\omega_{q} / 2 \pi=9.442 \mathrm{GHz}$, for six increasing values of the driving strength $(-66 \mathrm{dBm}$, green $\circ ;-52 \mathrm{dBm}$, blue $\triangle ;-49 \mathrm{dBm}$, orange $\Delta ;-46 \mathrm{dBm}$, yellow $\square ;-41 \mathrm{dBm}$, pink •; and $-36 \mathrm{dBm}$, brown *) superimposed on top of the theoretical predictions of the GJC model with four levels (shown in solid black lines). (b) Cavity transmission as a function of the driving frequency for all the considered theoretical models: GJC in solid blue, JC in dashed green, its Duffing reduction in dotdashed red, the effective Fokker-Planck model in orange with full circles for the experimental transmon anharmonicity coefficient $\chi / 2 \pi=-150 \mathrm{MHz}$, and a dot-dashed line for $\chi / 2 \pi=$ $-20 \mathrm{MHz}$, alongside the experimental data in yellow $\square$ 's for the driving power of $-46 \mathrm{dBm}$. It is clear that (only) the GJC model shows excellent agreement with the experimental data.

progressively in a measurement of complex amplitude, is due to the phase differences between the two metastable states. In the experimental response, we can also discern a split of the main peak, alluding to dynamical Rabi splitting [12]. The position of the dip shifts to the lower frequencies with increasing drive strength, while the split gradually fades away in favour of a Duffing-type profile.

In order to gain a further insight, we undertake an analytical approach by identifying an effective Hamiltonian to produce a (second-order) Fokker-Planck equation (FPE) for the transmon, following the adiabatic elimination of the cavity. FPEs in the generalized $P$ representation can be used to solve exactly for the steady state of quantum systems subject to the so-called potential conditions and have been used to study single nonlinear resonator systems [6]. For our two-oscillator model, these conditions are not satisfied, yet in the limit $2 \kappa \gg \gamma, \gamma_{\phi}$, the cavity can be eliminated in a similar fashion to the method of Ref. [44]. This process leaves a FPE for an effective one-oscillator system, which resembles a driven, damped quantum Duffing oscillator with anharmonicity $\chi$ [27] but with parameters that are nontrivial functions of those of the full system. Full details of this method can be found in Ref. [45]. The first moment of the cavity field in the steady state is

$$
\langle a\rangle=\frac{2}{\tilde{\gamma}_{c}}\left(\varepsilon_{d}-\frac{\tilde{\varepsilon} g}{\chi c} \frac{{ }_{0} F_{2}\left(c+1, c^{*} ; 2\left|\frac{\tilde{\varepsilon}}{\chi}\right|^{2}\right)}{{ }_{0} F_{2}\left(c, c^{*} ; 2\left|\frac{\tilde{\varepsilon}}{\chi}\right|^{2}\right)}\right),
$$

where ${ }_{0} F_{2}(x, y ; z)$ is a generalized hypergeometric function, and we have defined effective decay constants for the cavity $\tilde{\gamma}_{c}=\kappa+2 i \Delta \omega_{c}$ and transmon $\tilde{\gamma}_{q}=\gamma+2 i \Delta \omega_{q}+2 g^{2} / \tilde{\gamma}_{c}$, respectively (with $\Delta \omega_{c(q)}=\omega_{c(q)}-\omega_{d}$ ), effective drive strength $\tilde{\varepsilon}=-2 i g \varepsilon_{d} / \tilde{\gamma}_{c}$, and also $c=\tilde{\gamma}_{q} /(2 i \chi)$. The calculated transmission amplitude via Eq. (4) is plotted in Fig. 3(b) and compared to the exact ME results alongside the experimental data. The effective Fokker-Planck model exaggerates the actual nonlinearity in this regime, yet a lower value of $\chi$ allows us to capture the essential features of the full transmon-cavity-driven interaction (more details in Ref. [39]).

Discussion and concluding remarks.-We have examined the dispersive interaction of a single qubit and a microwave cavity mode, tracking nonlinearity with increasing drive power. When the regime of bistability is reached, simultaneous switching events allow for both of the metastable states to participate even at zero temperature. Their different phases cause the dip in coherent transmission, for which we have presented theoretical and experimental evidence. Interestingly, the dim quasicoherent state is preceded by a lower amplitude nonclassical state which is not predicted by the mean-field treatment. This state is characterized by very low photon numbers and intense fluctuations in the qubit inversion, which occupies now the north pole of the Bloch sphere. For high excitations, beyond the Duffing oscillator regime, both the cavity and the qubit participate in the switching, and the quantitative comparison with the experiment necessitates the inclusion of more than two levels of the transmon. The superconducting devices we have considered serve as examples of quantum activation with more than one quantum oscillator. 
The data underlying this work is available without restriction [46].

Th. K. M. wishes to thank H. J. Carmichael and L. Riches for instructive discussions. Th. K. M. and M. H. S. acknowledge support from the Engineering and Physical Sciences Research Council (EPSRC) under Grants No. EP/ I028900/2 and No. EP/K003623/2. E. G. acknowledges support from the EPSRC under Grant No. EP/L026082/1. P.J.L. acknowledges support from the EPSRC under Grants No. EP/J001821/1 and No. EP/M013243/1.

*t.mavrogordatos@ucl.ac.uk

[1] J. Raimond, M. Brune, and S. Haroche, Rev. Mod. Phys. 73, 565 (2001).

[2] J. Koch, T. M. Yu, J. Gambetta, A. A. Houck, D. I. Schuster, J. Majer, A. Blais, M. H. Devoret, S. M. Girvin, and R. J. Schoelkopf, Phys. Rev. A 76, 042319 (2007).

[3] I. Chiorescu, P. Bertet, K. Semba, Y. Nakamura, C. J. P. M. Harmans, and J. E. Mooij, Nature (London) 431, 159 (2004).

[4] A. Wallraff, D. I. Schuster, A. Blais, L. Frunzio, R.-S. Huang, J. Majer, S. Kumar, S. M. Girvin, and R. J. Schoelkopf, Nature (London) 431, 162 (2004).

[5] Y. Nakamura, Y. A. Pashkin, and J. S. Tsai, Nature (London) 398, 786 (1999).

[6] D. F. Walls and G. J. Milburn, Quantum Optics (Springer, Berlin, 2010).

[7] M. Aspelmeyer, T. J. Kippenberg, and F. Marquardt, Rev. Mod. Phys. 86, 1391 (2014).

[8] H. J. Carmichael, Phys. Rev. X 5, 031028 (2015).

[9] G. Rempe, R. J. Thompson, R. J. Brecha, W. D. Lee, and H. J. Kimble, Phys. Rev. Lett. 67, 1727 (1991).

[10] J. Kerckhoff, M. A. Armen, and H. Mabuchi, Opt. Express 19, 24468 (2011).

[11] L. Tian and H. J. Carmichael, Phys. Rev. A 46, R6801 (1992).

[12] L. S. Bishop, J. M. Chow, J. Koch, A. A. Houck, M. H. Devoret, E. Thuneberg, S. M. Girvin, and R. J. Schoelkopf, Nat. Phys. 5, 105 (2009).

[13] R. Vijay, M. H. Devoret, and I. Siddiqi, Rev. Sci. Instrum. 80, 111101 (2009).

[14] H. J. Carmnichael, Statistical Methods in Quantum Optics 2 (Springer, New York, 2008).

[15] K. W. Murch, E. Ginossar, S. J. Weber, R. Vijay, S. M. Girvin, and I. Siddiqi, Phys. Rev. B 86, 220503(R) (2012).

[16] M. Boissonneault, J. M. Gambetta, and A. Blais, Phys. Rev. Lett. 105, 100504 (2010).

[17] L. S. Bishop, E. Ginossar, and S. M. Girvin, Phys. Rev. Lett. 105, 100505 (2010).

[18] M. D. Reed, L. DiCarlo, B. R. Johnson, L. Sun, D. I. Schuster, L. Frunzio, and R. J. Schoelkopf, Phys. Rev. Lett. 105, 173601 (2010).

[19] M. Dykman and V. N. Smelyanskii, Sov. Phys. JETP 67, 1769 (1988).

[20] M. I. Dykman, Fluctuating Nonlinear Oscillators (Oxford University Press, New York, 2012).

[21] V. Peano and M. Thorwart, Europhys. Lett. 89, 17008 (2010).

[22] A Kamenev, Field Theory of Non-equilibrium Systems (Cambridge University Press, Cambridge, England, 2011).
[23] R. S. Maier and D. L. Stein, Phys. Rev. Lett. 69, 3691 (1992).

[24] M. I. Dykman, M. M. Millonas, and V. N. Smelyanskiy, Phys. Lett. A 195, 53 (1994).

[25] R. Graham and T. Tél, Phys. Rev. Lett. 52, 9 (1984).

[26] H. Paik et al., Phys. Rev. Lett. 107, 240501 (2011).

[27] P. D. Drummond and D.F. Walls, J. Phys. A 13, 725 (1980).

[28] Here, we denote the photon loss rate by $2 \kappa$, in alignment with the rate equations of laser theory.

[29] P. Carbonaro, G. Compagno, and F. Persico, Phys. Lett. 73A, 97 (1979).

[30] This expansion brings us to the fundamental $\sqrt{N}$ nonlinearity of the driven JC oscillator [31] with $N$ excitations. The oscillator response has been studied more extensively at resonance, where single-atom absorptive optical bistability was first reported in Ref. [32]. The $\sqrt{N}$ anharmonic oscillator is invoked [33] alongside two excitation ladders, each associated with a bosonic field [14]. Strong driving leads to spontaneous symmetry breaking and phase bistability following a second-order quantum phase transition [34]. In the dispersive regime, on the other hand, the $\sqrt{N}$ eigenvalue dependence allows for a perturbation expansion in powers of $N / N_{\text {crit }}$, where $N_{\text {crit }}=\delta^{2} /\left(4 g^{2}\right)$ is a characteristic system parameter, indicating the driving region where nonlinearity is important. The expansion is carried out around a critical point in the mean-field phase diagram [17].

[31] J. M. Fink, M. Göppl, M. Baur, R. Bianchetti, P. J. Leek, A. Blais, and A. Wallraff, Nature (London) 454, 315 (2008).

[32] C. M. Savage and H. J. Carmichael, IEEE J. Quantum Electron. 24, 1495 (1988).

[33] Y. T. Chough and H. J. Carmichael, Phys. Rev. A 54, 1709 (1996).

[34] P. Alsing and H. J. Carmichael, Quantum Opt. 3, 13 (1991).

[35] The drive terms can also be shown to remain linear in the cavity operators for low enough drives [17].

[36] A. Blais, R. S. Huang, A. Wallraff, S. M. Girvin, and R. J. Schoelkopf, Phys. Rev. A 69, 062320 (2004).

[37] H.-P. Breuer and F. Petruccione, The Theory of Open Quantum Systems (Oxford University Press, New York, 2002).

[38] P. E. Kloeden and E. Platen, Stochastic Modelling and Applied Probability (Springer, New York, 1995).

[39] See Supplemental Material at http://link.aps.org/ supplemental/10.1103/PhysRevLett.118.040402, which includes Refs. [40-41], for details on the experimental setup as well as additional theoretical support regarding various aspects of simultaneous bistability, with the same methods employed as in the main text, alongside a further comparison between theory and experiment.

[40] R. Bonifacio, M. Gronchi, and L. A. Lugiato, Phys. Rev. A 18, 2266 (1978).

[41] H. J. Carmnichael, Statistical Methods in Quantum Optics 1 (Springer, New York, 1999).

[42] E. Ginossar, L. S. Bishop, D. I. Schuster, and S. M. Girvin, Phys. Rev. A 82, 022335 (2010).

[43] O. V. Zhirov and D. L. Shepelyansky, Phys. Rev. Lett. 100, 014101 (2008).

[44] P. D. Drummond, K. J. McNeil, and D. F. Walls, Opt. Acta 28, 211 (1981).

[45] M. Elliott and E. Ginossar, Phys. Rev. A 94, 043840 (2016).

[46] DOI: 10.15126/surreydata.00813140. 